\title{
ARTICLE Early-life high-fat diet-induced obesity programs hippocampal development and cognitive functions via regulation of gut commensal Akkermansia muciniphila
}

\author{
Youjun Yang ${ }^{1}$, Zhanqiong Zhong ${ }^{1}$, Baojia Wang ${ }^{1}$, Xiuwen Xia ${ }^{1}$, Weiyi Yao ${ }^{1}$, Ling Huang ${ }^{1}$, Yili Wang ${ }^{1}$ and Weijun Ding ${ }^{1}$
}

\begin{abstract}
Obesity is one of the most serious public health challenges in the world. Obesity during early life has been associated with an increased risk of neurodevelopmental disorders, including deficits in learning and memory, yet the underlying mechanisms remain unclear. Here, we show that early life high-fat diet (HFD) feeding impairs hippocampus-dependent contextual/spatial learning and memory, and alters the gut microbiota, particularly by depleting Akkermansia muciniphila (A. muciniphila), in mice. Transplantation of the HFD microbiota confers hippocampus-dependent learning and memory deficits to mice fed a chow diet. Oral treatment of HFD-fed mice with the gut commensal A. muciniphila corrects gut permeability, reduces hippocampal microgliosis and proinflammatory cytokines (tumor necrosis factor- $\alpha$ (TNF- $\alpha$ ), interleukin-1 $\beta$ (IL-1 $\beta$ ) and IL-6) expression, and restores neuronal development and synapse plasticity, thus ameliorates defects in learning and memory. Interestingly, treatment of mice with lipopolysaccharide (LPS) mimics HFD-induced hippocampus-dependent cognitive impairment in chow-fed mice. In line with these findings, pharmacologic blockade of Toll-like receptor 4 (TLR4) signalling or antibiotics treatment both effectively prevent hippocampus-dependent learning and memory deficits in HFD-fed mice. Collectively, our findings demonstrate an unexpected pivotal role of gut microbiota in HFD-induced cognitive deficits and identify a potential probiotic therapy for obesity associated with cognitive dysfunction during early life.
\end{abstract}

Neuropsychopharmacology (2019) 44:2054-2064; https://doi.org/10.1038/s41386-019-0437-1

\section{INTRODUCTION}

Childhood and adolescent obesity is considered a tremendous public health challenge in the world. In addition to its broad impact on cardiovascular and metabolic health, compelling studies suggesting an association between obesity and cognitive deficits [1-5], such detrimental effects have been found in obese and overweight people across their whole lifespan, suggesting that excess adiposity earlier in life may not only increase risk for later dementia, but also may be associated with some extent of cognitive dysfunction in youth long prior to dementia onset $[5,6]$.

Previous studies have shown that high-fat diet (HFD) reduces the expression of intestinal tight junction protein and disrupted gut barrier integrity, leading to translocation of bacterial lipopolysaccharide (LPS) into the blood and producing inflammation [7]. Interestingly, LPS-induced inflammation can produce both reversible cognitive deficits and acute brain injury contributing to long-term cognitive impairment by interleukin-1 (IL-1)-dependent mechanisms [8]. Microglia, the mononuclear phagocytes of the central nervous system (CNS), are important for the maintenance of CNS homeostasis, but also critically contribute to CNS pathology, microglial activation has been associated with synapse loss, and cognitive dysregulation [9-11]. Previous study demonstrated that HFD-induced obesity alters microglial morphology, diminishes synaptic markers, and impairs cognitive function [2]. Microglia express numerous members of the Toll-like receptor (TLR) family, which recognize conserved microbial motifs expressed by a wide array of pathogens. For example, LPS binds to TLR4 and activates c-Jun N-terminal kinase (JNK) and nuclear factor-kappa B (NF-KB), two important inflammatory signaling molecules. The activation of TLR4-JNK/NF-KB signaling pathway leads to the production of proinflammatory cytokines, such as tumor necrosis factor- $\alpha$ (TNF- $\alpha$ ), IL-1 $\beta$, and IL- 6 , and contributes to the development of neurodegenerative diseases [12-14]. Hippocampal neurogenesis and its related learning and memory are influenced negatively by microglial activation and neuroinflammation [15-17]. Obesity is characterized by low-grade systemic inflammation, which is associated with an alteration of gut microbiota $[4,18,19]$. The composition and function of the gut microbiota is significantly shaped by diet. Diet-induced alteration in the gut microbiota are persistent and reproducible, and as such, have lasting effects on the host [7, 20]. Evidence has linked a HFD with alterations of gut microbiota and cognitive impairment [18]. However, we still lack a full understanding of the mechanisms by which microbiota may influence the gut-brain axis and, thereby, brain function and behavior during obesity.

Recently, the gut commensal Akkermansia muciniphila (A. muciniphila) has been identified as a mucin-degrading bacteria that resides in the mucus layer [21-23]. A. muciniphila represent $3-5 \%$ of the microbial community in healthy subjects, and its abundance inversely correlates with body weight and type 1 diabetes in mice and humans $[24,25]$. A purified membrane protein from A. muciniphila or the pasteurized bacterium improves metabolism in obese and diabetic

\footnotetext{
${ }^{1}$ School of Basic Medical Sciences, Chengdu University of Traditional Chinese Medicine, 1166 Liutai Road, 611137 Chengdu, China
} Correspondence: Youjun Yang (youjunyang@email.ncu.edu.cn) 
mice [26]. A. muciniphila treatment reverses HFD-induced metabolic disorders, including fat-mass gain, metabolic endotoxemia, adipose tissue inflammation, insulin resistance [20], and increases the intestinal levels of endocannabinoids that control inflammation, the gut barrier and gut peptide secretion [20]. Besides, A. muciniphila treatment ameliorates alcoholic liver disease [27], controls islet autoimmunity in nonobese diabetic (NOD) mice [28], mediates the antiseizure effects of the ketogenic diet [29], and orchestrates energy homeostasis during cold [30].

Here we found that early life HFD-induced obesity in mice is associated with hippocampus-dependent learning and memory deficits, which are mediated by alterations in the gut microbiome. Notably, we also reported that HFD-induced changes in the gut microbiota block neuronal development and plasticity in the hippocampus. Moreover, oral treatment with gut commensal $A$. muciniphila restores intestinal permeability, microgliosis, neurodevelopment, and neuronal plasticity in the hippocampus of HFDfed mice through correcting gut permeability, thus preserving cognitive function. Our findings suggest that targeting the microbiome may represent an approach for targeting the individual with obesity-related cognitive deficits.

\section{MATERIALS AND METHODS}

Details regarding protein extraction and western blotting, immunohistochemistry and confocal microscopy, 5-bromo-2deoxyuridine (BrdU) assays, brain slice electrophysiology, Golgi impregnation and tracing, qRT-RNA analysis, fecal DNA extraction and $16 \mathrm{~S}$ ribosomal RNA (rRNA) gene sequencing, fecal microbial transplantation, antibiotic treatment, LPS injection, resatorvid (TAK-242) and LPS-RS administration, and intestinal permeability assay are available in the Supplemental materials and methods.

\section{Mice and diet}

Juvenile C57BL/6J male mice (postnatal day 21, P21) were obtained from Chengdu DaShuo Experimental Animal Company and were kept on a $12 \mathrm{~h}$ light/dark cycle with access to food and water ad libitum in a temperature-controlled room $\left(25^{\circ} \mathrm{C}\right)$. All mice were maintained in a light and humidity controlled climatic chamber (three mice/chamber) for the SPF conditions and fed with HEPA-filtered air, and provided with irradiated food and water. Mice were placed on either a regular chow diet (chow) (Lab Diets, \#5053) or HFD consisting of $60 \%$ kcal from fat, $20 \% \mathrm{kcal}$ from protein, and $20 \% \mathrm{kcal}$ from carbohydrates (Research Diets, \#D12492). Weight was measured weekly, fat mass were measured using an NMR (Minispec body composition analyzer, Bruker Optics, Billerica, MA). After 6 weeks on diet, mice were conducted to behavior test or tissue collection. All animal procedures were carried out in strict accordance with the principles of laboratory animal care and use approved by the Chengdu University of TCM Animal Care and Use Committee guidelines.

\section{Contextual fear-conditioning test}

Contextual fear-conditioning test were carried out in accordance with previously established behavioral schemes with slight modifications [31]. Briefly, mice were fear-conditioned in a test cage inside a sound-attenuated box. On conditioning day, test mice were placed in the box and allowed to explore for $2 \mathrm{~min}$ for habituation. After habituation, mice were conditioned with five or ten trials consisting of a 30 -s tone $(5 \mathrm{kHz}, 70 \mathrm{~dB})$ and received a $2 \mathrm{~s}, 0.5 \mathrm{~mA}$ foot shock delivered through the electrified floor grid. Each trial was separated by a $30 \mathrm{~s}$ inter-trial interval. After the final tone-shock pairing, mice remained in the conditioning chamber for $1 \mathrm{~min}$ before being returned to their home cages. Trials were videotaped, and freezing during the initial 2 -min acclimation/exploration period was scored as a measure of baseline freezing to the conditioning context. Memory for the context was assessed after specified time intervals by returning the mice to contextual box, where freezing behavior was scored during the last $3.5 \mathrm{~min}$ of the total $5.5 \mathrm{~min}$ spent in the chamber. After the contextual fear test, mice were returned to their home cages. Memory for the cue was assessed by placing the mice in a novel box. After a 2 min acclimation period in novel box, mice were presented with three $30-\mathrm{s}$ tones $(5 \mathrm{kHz}, 70 \mathrm{~dB})$ separated by an inter-trial interval of $30 \mathrm{~s}$. Freezing behavior was scored during each of the 30-s tone presentations. The freezing time of the mouse was measured by Video Fear Conditioning "Video Freeze ${ }^{\circledR "}$ Software (SOF-843, Med Associates).

\section{Barnes circular maze test}

Barnes circular maze test were carried out in accordance with previously established behavioral schemes with slight modifications [32]. Briefly, animals were tested during light cycle and were transferred to the behavior testing room with light control ( $80 \mathrm{Lx}$ ) for $1 \mathrm{~h}$ before the start of experiments. Spatial cues with distinct patterns and shapes were placed on the wall of the testing room. A 500 lux light was turned on during the trial. One day before the training trials began, test mice were habituated in the target box for $3 \mathrm{~min}$. The training trials were repeated for 4 consecutive days, and three trials were performed each day with $20 \mathrm{~min}$ inter-trial intervals. The movement of the mouse was recorded, and the number of errors made, and the latency to find the target hole were recorded during the training trials by video tracking software (BMT-100, Chengdu Techman Software, China). On day 5, the probe trial was performed with each mouse, the escape box was removed, the test mouse was allowed to find the target hole freely for $90 \mathrm{~s}$, and the time in the target area was measured.

Bacterial culture and treatment

A. muciniphila (ATCC BAA845) was cultured under anaerobic conditions at $37^{\circ} \mathrm{C}$ in Brain Heart Infusion media supplemented with $0.05 \%$ hog gastric mucin type III (Sigma-Aldrich) [29]. L. reuteri MM4-1A (ATCC-PTA-6475) was cultured anaerobically in MRS broth in a $90 \% \mathrm{~N}_{2}, 5 \% \mathrm{CO}_{2}$, and $5 \% \mathrm{H}_{2}$ environment [33]. $A$. muciniphila and $L$. reuteri were freshly cultured in anaerobic conditions as described above, and then washed, pelleted, and resuspended at $5 \times 10^{9} \mathrm{cfu} / \mathrm{ml}$ in prereduced PBS. For heat killing, A. muciniphila were placed at $100^{\circ} \mathrm{C}$ for $20 \mathrm{~min}$. Mice were gavaged every $12 \mathrm{~h}$ for 28 days with $200 \mu \mathrm{l}$ bacterial suspension or sterile prereduced PBS as vehicle control.

Measurement of hippocampal cytokines and serum endotoxin Hippocampal tissue homogenates were prepared in RIPA buffer containing protease inhibitor cocktail (ThermoFisher) and diluted into PBS. TNF- $a, I L-1 \beta$ and IL- 6 ELISAs (eBioscience) were performed according to manufacturer's instructions. Serum endotoxin was quantified using a Limulus amaebocyte lysate kit (Cambrex Bio Science) according to the manufacturer's instructions. Briefly, serum samples were diluted 1:10 to 1:1000 in endotoxin-free water (Lonza), heated at $70^{\circ} \mathrm{C}$ for $10-15 \mathrm{~min}$ and processed according to the manufacturer's protocol. Recovery rate was determined based on the net LPS concentration of spiked samples supplemented with LPS $(0.1 \mathrm{EU} / \mathrm{mL}$, SigmaAldrich). Samples with a recovery rate of at least $50 \%$ were considered.

\section{Statistical analyses}

The investigators were blinded to allocation during experiments and outcome assessment for behavioral analyses, voltage-clamp recordings, and analysis of hippocampal patches. No animals or data points were excluded from analyses. Results are expressed as mean \pm SEM. For behavioral experiments, statistics were based on the two-sided unpaired Student's $t$-tests, one- or two-way ANOVA with Bonferroni post hoc analysis to correct for multiple comparisons, unless otherwise indicated. $P, t$, and $F$ values are presented in 

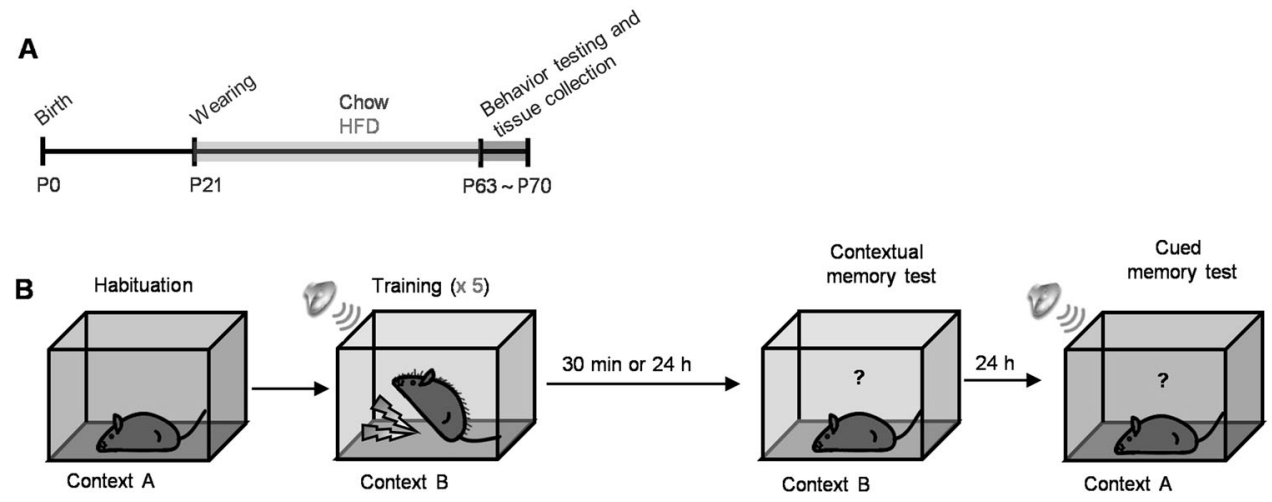

C

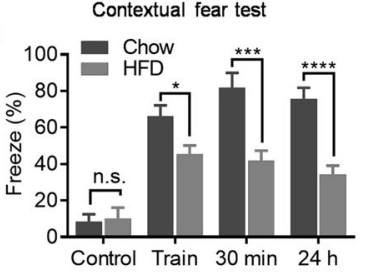

F
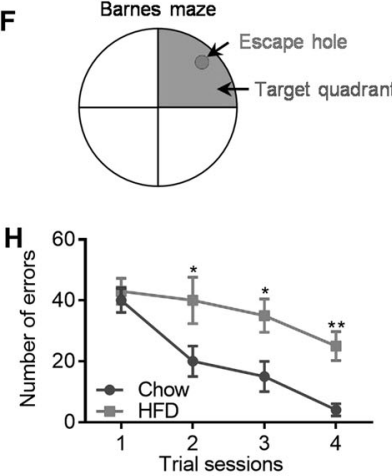

L
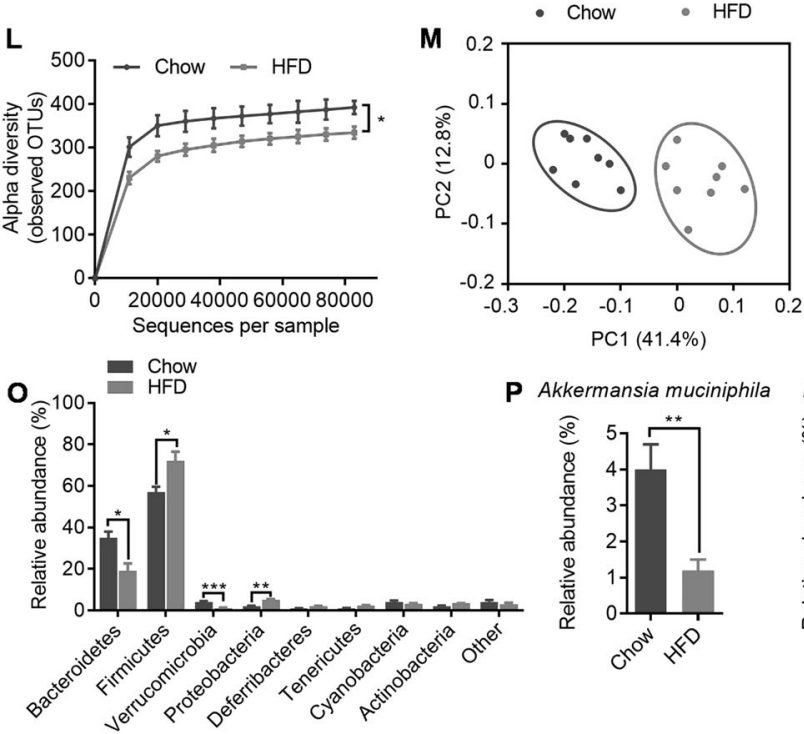

P Akkermansia muciniphila
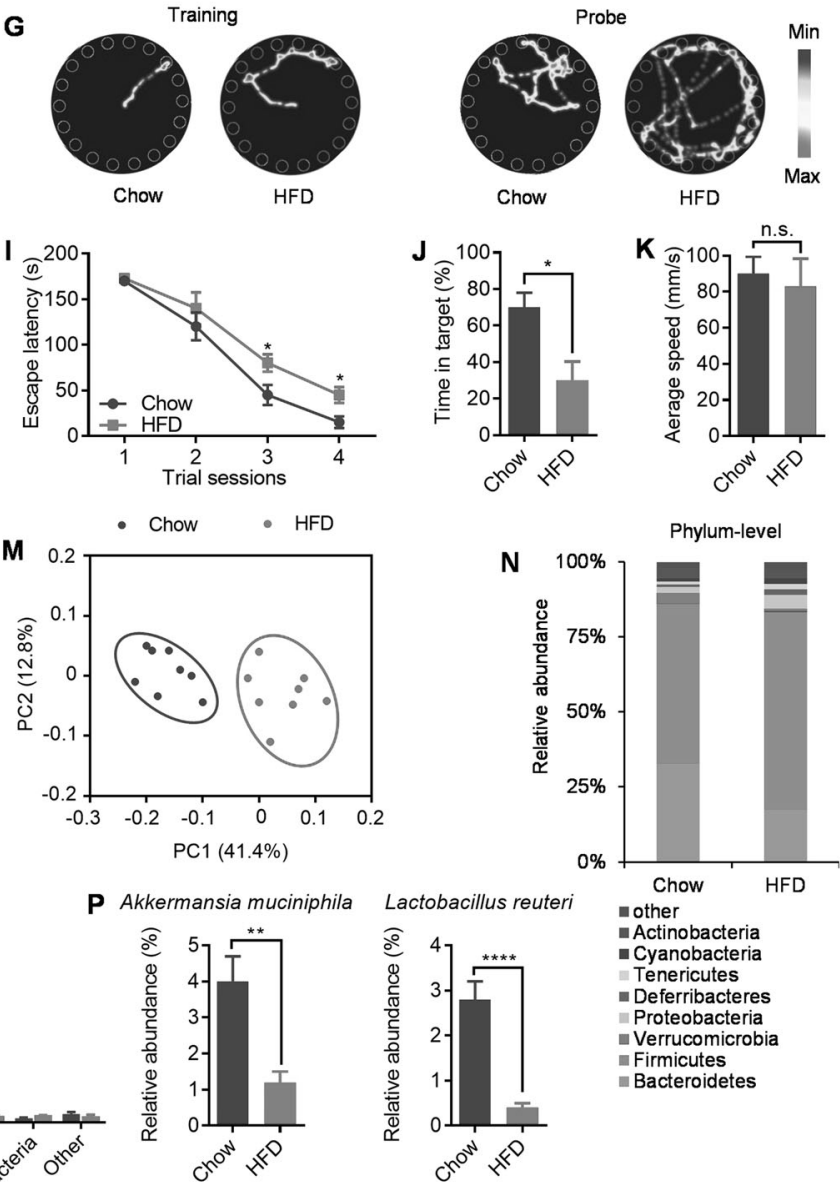

Phylum-level

$$
]_{1.2}
$$

$\mathbf{N}$
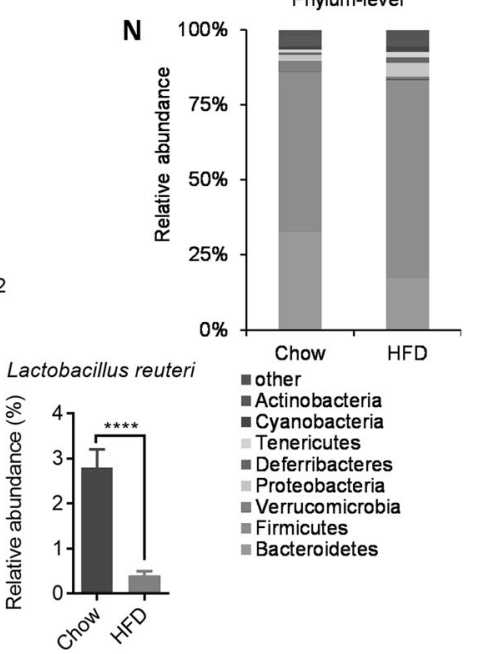

- Actinobacteria - Cyanobacteria Tenericutes

- Deferribacteres

Proteobacteria

Verrucomicrobia

- Firmicutes

Bacteroidetes

the Figure legends, $n$ values are provided in the Figures. $p<0.05$ was considered significant. ${ }^{*} p<0.05,{ }^{* *} p<0.01,{ }^{* * *} p<0.001$, and ${ }^{* * * *} p<$ 0.0001 . GraphPad Prism 6 (GraphPad Software) and IBM SPSS Statistics 20 (St Leonards) software were used to perform statistical analyses and for generating graphical representations of data.

\section{RESULTS}

Hippocampus-dependent contextual/spatial learning and memory are impaired in early life HFD-fed mice

To investigate how HFD-induced obesity affects neurodevelopment and cognitive function, juvenile male mice were fed either 
Fig. 1 Cognitive functions deficits and dysbiosis of the gut microbiota in HFD mice. a Schematic of chow and HFD feeding experiment. $\mathbf{b}$ Experimental design for contextual and cued fear-conditioning test. $\mathbf{c}$ Percent of freezing in the contextual fear-conditioning test. $\mathbf{d}$ Percent of freezing in cued fear conditioning test. e Experimental design for Barnes circular maze test. f Barnes circular maze. Arrows showed the position of escape hole and target quadrant. g Representative traces of chow- and HFD-fed mice in the Barnes circular maze test during training and probe trial. $\mathbf{h}$ Number of errors in the training session of Barnes circular maze. $\mathbf{i}$ Escape latency in the training session of Barnes circular maze test. $\mathbf{j}$ Time in the target quadrant during the probe trial on the fifth day of Barnes circular maze test. $\mathbf{k}$ Average speed during the probe trial on the fifth day of Barnes circular maze test. I Alpha diversity of fecal 16S rDNA sequencing data from mice fed chow or HFD for 6 weeks. $\mathbf{m}$ Principal coordinates analysis (PCOA) based on weighted UniFrac analysis of OTUs. Each symbol represents a single sample of feces after 6 weeks of chow feeding or HFD feeding. $\mathbf{n}$, o Comparison of phylum-level proportional abundance of feces sample after 6 weeks of chow or HFD feeding. $\mathbf{p}$ Relative abundances of $A$. muciniphila and L. reuteri in chow- and HFD-fed mice. $n=8 /$ group, means \pm SEM (error bars) are plotted. Statistics were performed with Student's $t$-test or one-way analysis of variance (ANOVA). ${ }^{*} P<0.05,{ }^{* *} P<0.01,{ }^{* * *} P<0.001$, and ${ }^{* * * *} P<$ 0.0001 , n.s., not significant

chow or HFD for 6 weeks after weaning (P21) (Fig. 1a). As expected, HFD-fed mice maintained higher bodyweights, higher body fat percentages, higher fat pad weight, and lower lean-bodymass percentages when compared with chow-fed littermates (Fig. S1a-d). Given that obesity has been associated with increased risk for neurodevelopmental disorders including cognitive impairment, we first employed contextual-fear conditioning test to evaluate contextual learning and memory in chow- and HFD-fed mice (Fig. 1b). Chow- and HFD-fed mice showed no difference in baseline freezing behavior during the habituation period (Fig. 1c). However, HFD-fed mice exhibited decreased freezing during fear conditioning and showed less freezing behaviors when returned to the shock cage $30 \mathrm{~min}$ and $24 \mathrm{~h}$ later when compared with chow-fed littermates (Fig. 1c). This indicates early life HFD feeding impairs hippocampus-dependent contextual learning and memory in mice. Interestingly, HFD feeding had a significant effect in the contextual fear test, but not cued fear test (Fig. 1d), suggesting a hippocampal-related, but not amygdala-related, change in HFD-fed mice [31]. There is a difference in the freezing percentage during the "training phase" between the chow- and HFD-fed mice, likely indicating a different sensitivity to the shock. Thus, we increased training sessions to achieve the same percent of freezing and then see whether memory deficits persist $30 \mathrm{~min}$ and $24 \mathrm{~h}$ later in HFD-fed mice (Fig. S2a, b). We found that HFD feeding resulted in a significant deficit in freezing responses when reexposure to the conditioning chamber after $30 \mathrm{~min}$ and $24 \mathrm{~h}$ (Fig. S2c), suggesting that deficits in contextual fear memory in HFD-fed mice were not due to altered locomotor activity or sensitivity.

To assess spatial learning and memory, we performed the Barnes circular maze test in which mice learn to rapidly escape a brightly lighted circular field by finding a specific dark hole at its periphery (Fig. 1e, f). Results revealed that HFD-fed mice were significantly impaired their learning performance during 4 days of training (Fig. 1g), with increased number of errors (Fig. 1h) and increased escape latency (Fig. 1i) to find the target hole when compared with chow-fed littermates. In the probe trials at day 5 , HFD-fed mice showed impaired memory performance, with decreased time in the target quadrant (Fig. 1g, j). However, there is no significant difference in the average speed between chowand HFD-fed mice (Fig. 1k). These data, together with the results of the contextual fear-conditioning test, indicated that early life HFD feeding severely impaired hippocampal-dependent learning and memory in mice, which are consistent with previous reports $[5,15,34]$.

HFD feeding alters the gut microbiota

To examine whether HFD induces alterations of gut microbiota, we analyzed the bacterial composition and community structure in the feces of chow- and HFD-fed mice by $16 \mathrm{~S}$ rRNA gene sequencing. The alpha diversity of microbiota, computed based on weighted UniFrac distances (the assessment of community structure by considering abundance of operational taxonomic units [OTUs]), in HFD-fed mice was reduced when compared to chow-fed mice (Fig. 11). These results indicated that the richness and diversity of gut microbial communities were decreased in HFD-fed mice. Principal coordinates analysis ( $P C O A)$ based on weighted UniFrac distance, showed major alterations of the microbiota content in feces samples of HFD-fed mice (Fig. 1m). As expected, the microbial communities in both chow- and HFD-fed mice were comprised of a typical mouse gut microbiota, dominated by Bacteroidetes and Firmicutes. However, we observed differences in OTUs abundance at phylum level in Bacteroidetes, Firmicutes, Verrucomicrobia, and Proteobacteria (Fig. 1n, o). Comparison of phylum level proportional abundance in feces showed shifts in proportions, especially in the ratio Firmicutes/Bacteroidetes. Firmicutes phylum increased its richness in feces up to $68.7 \%$ under HFD feeding (compared to $57.3 \%$ in chow-fed mice) and Bacteroidetes decreased it to $19.7 \%$ (compared to $36.8 \%$ in chow-fed mice) (Fig. 1n, o). When looking at the most significantly changed OTUs using analysis of variance, Akkermansia muciniphila (A. muciniphila) and Lactobacillus reuteri (L. reuteri) were the most reduced bacteria (Fig. 1p). Together, these results demonstrate a major shift in gut microbiota in response to HFD exposure, which are consistent with previous report [20].

Gut microbiota mediated HFD-induced hippocampal-dependent learning and memory deficits

To identify functional differences between gut microbial communities and determine whether their role is causal, we transplanted fecal microbiota from adult chow- and HFD-fed mice into 3-weekold antibiotics (Abx)-pretreated mice (Fig. 2a, fed with a regular chow diet). As expected, Abx treatment reduced the fecal bacterial load by $>2$ log-folds (Fig. S3a), decreased alpha diversity as measured by the Shannon index (Fig. S3b). Next, we characterized the bacterial gut microbiome of the reconstituted chow FTM mice and HFD FTM littermates. As shown in the PCoA in Fig. S3c, the gut microbiome of recipient mice clustered together with that of donor mice without major changes. Assessment of taxa abundance in chow, chow FTM, HFD, and HFD FTM mice confirmed the above observations at the rank of phylum (Fig. 3Sd). This indicates that the preservation and transfer protocol had no major effect on commensal community structure, confirming engraftment efficacy. Interestingly, Abx-pretreated mice that received fecal microbiota from chow-fed mice showed normal hippocampaldependent cognitive function (Fig. 2b-g). By contrast, hippocampal-dependent cognitive function was impaired in Abx-pretreated mice that received fecal microbiota from HFDfed mice (Fig. $2 b-g$ ). Taken together, these data revealed that gut microbiome play a critical role in HFD-induced hippocampaldependent cognitive deficits.

Since $A$. muciniphila treatment could reverse HFD-induced metabolic disorders, including fat-mass gain, metabolic endotoxemia, adipose tissue inflammation, and insulin resistance $[20,24-26]$. We hypothesized that the most decreased $A$. muciniphila in the microbiota of HFD mice was causally related to their cognitive deficits. To investigate whether this strain alone 

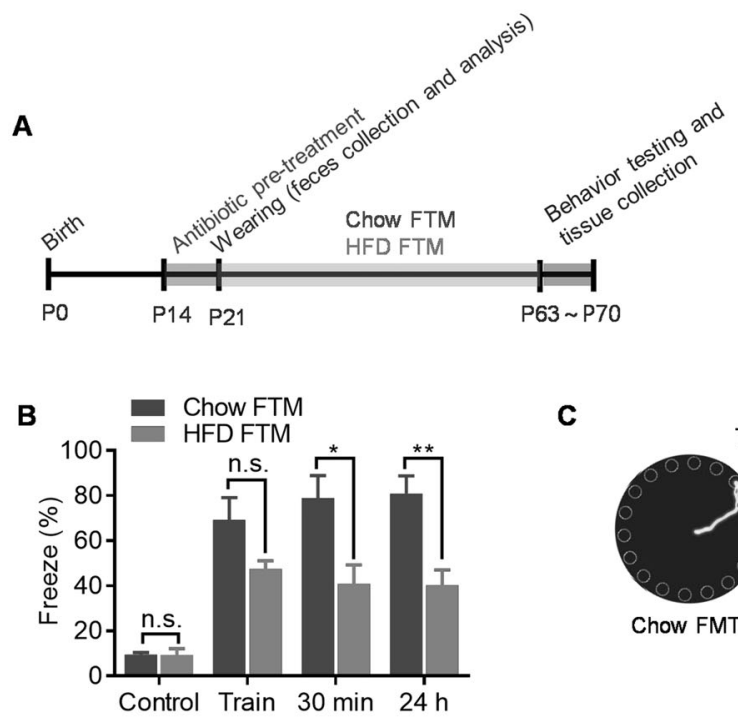

C
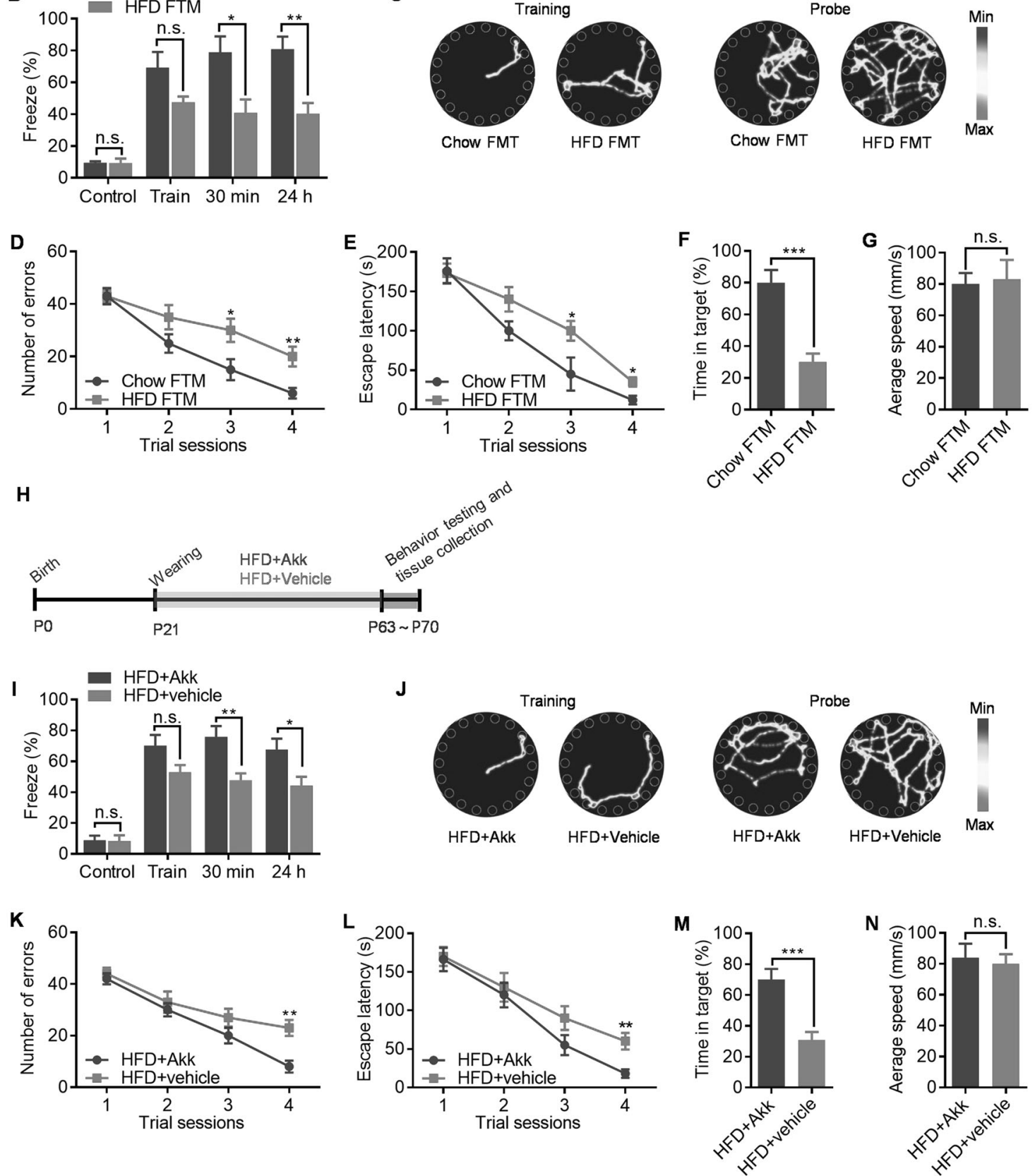

could revert part of the HFD-induced phenotype, we reintroduced A. muciniphilas into HFD-fed mice at weaning for 6 weeks, after which behavior was tested (Fig. 2h). Remarkably, treatment with $A$. muciniphilas significantly improved hippocampal-dependent cognitive performance in HFD-fed mice (Fig. 2i-n). However, addition of $A$. muciniphila had no major effect on bacterial composition of HFD-fed mice (Fig. S3c, d). Results from several control experiments underscore the specificity of $A$. muciniphilas-mediated rescue of hippocampal-dependent cognitive function in HFD-fed mice. First, treatment with heat-killed $A$. muciniphilas $\left(100^{\circ} \mathrm{C}\right.$ for 
Fig. 2 HFD-associated microbiota impairs cognitive function in antibiotics pretreated mice fed a regular chow diet due to absence of $A$. muciniphilas. a Schematic of chow FTM and HFD FTM experiment. b Percent of freezing in the contextual fear-conditioning test. c Representative traces of chow FTM mice and HFD FTM mice in the Barnes circular maze test. $\mathbf{d}$ Number of errors in the training session of Barnes circular maze. e Escape latency in the training session of Barnes circular maze test. $\mathbf{f}$ Time in the target quadrant during the probe trial on the fifth day of Barnes circular maze test. $\mathbf{g}$ Average speed during the probe trial on the fifth day of Barnes circular maze test. $\mathbf{h}$ Schematic of $A$. muciniphilas reintroduction experiment. $\mathbf{i}$ Percent of freezing in the contextual fear-conditioning test. $\mathbf{j}$ Representative traces of $A$. muciniphilas- and vehicle-treated mice in the Barnes circular maze test. k Number of errors in the training session of Barnes circular maze. I Escape latency in the training session of Barnes circular maze test. $\mathbf{m}$ Time in the target quadrant during the probe trial on the fifth day of Barnes circular maze test. $\mathbf{n}$ Average speed during the probe trial on the fifth day of Barnes circular maze test. $n=8 / \mathrm{group}$, means \pm SEM (error bars) are plotted. Statistics were performed with Student's $t$-test or one-way analysis of variance (ANOVA). ${ }^{*} P<0.05,{ }^{* *} P<0.01$, and ${ }^{* * *} P<0.001$, n.s., not significant

$20 \mathrm{~min}$ ) failed to restore hippocampal-dependent cognitive function in HFD-fed mice (Fig. S4a-g). Second, similar treatment with another bacteria, $L$. reuteri, whose abundance is also reduced in the gut microbiota of HFD-fed mice, failed to rescue hippocampal-dependent cognitive function in HFD-fed mice (Fig. S4h-n). Besides, A. muciniphilas-treated mice maintained lower bodyweights and had lower body fat percentages and higher lean-body-mass percentages when compared with vehicletreated littermates (data not shown), which is consistent with previous report [20]. Overall, these findings reveal that $A$. muciniphilas specifically rescues hippocampal-dependent cognitive function in HFD-fed mice.

Treatment with $A$. muciniphilas reverses both hippocampal neuronal development and long-term potentiation (LTP) in HFDfed mice

Some forms of cognitive function deficits are postulated to be caused by disruption of synaptic transmission between excitatory and inhibitory circuits. Thus, we recorded, in whole-cell configuration, miniature inhibitory and miniature excitatory postsynaptic currents (mIPSC and mEPSC, respectively) of pyramidal neurons in the hippocampal CA1 region. When action potentials were blocked with tetrodotoxin (TTX, $1 \mathrm{mM}$ ), recordings of mIPSC from the hippocampal $\mathrm{CA} 1$ region showed that amplitude and frequency were not altered, indicating normal inhibitory synaptic function in HFD-fed mice (Fig. 3a-c). Similarly, in the presence of TTX, the frequency of mEPSC was unchanged (Fig. 3d, e). However, the amplitude of mEPSC in hippocampal CA1 from HFD-fed mice was reduced with a clear right shift in the cumulative distribution of mEPSC inter-event intervals (Fig. 3d, f). Consistent with this, LTP in the hippocampus was impaired in HFD-fed mice (Fig. $3 g-i$ ). Therefore, these results indicate that HFD do not affect the formation of inhibitory synapses or function on CA1 pyramidal neurons during early postnatal development but impair the development of normal excitatory synaptic function and synaptic plasticity in the hippocampus.

We then characterized the neuronal proliferation in the hippocampal dentate gyrus (DG). Using BrdU labeling, we found decreased neuronal proliferation in P35 and P63 (adult) in HFDfed mice, but not at P21 (Fig. 3j, k). Another neuronal characteristic that can also be correlated with cognitive performance is dendritic morphology and complexity. GolgiCox staining and Sholl analysis of the dendritic length of DG granule cells of chow- and HFD-fed mice revealed that the proximal quarter of the arbor (between 0 and $60 \mu \mathrm{m}$ from the neuron) was similar (Fig. 3l, m). However, neurons from HFD-fed mice exhibited shorter dendritic length in distal areas $(60-240 \mu \mathrm{m})$ (Fig. 3l, m), and less spine density (Fig. 3n, o) when compared with chow-fed littermates. A reduction in AMPA receptor and/or NMDA receptor (AMPAR and/or NMDAR) expression could account for the LTP deficit in HFD-fed mice. We therefore, examined protein levels in total, postsynaptic density (PSD) hippocampal fractions. We found that the expression of GluA1 and GluA2 subunits was substantially reduced in both total hippocampal lysate and PSD fraction from the HFD-fed mice (Fig. 3p, q). In contrast, protein levels of NR2A and NR2B subunits in the total lysate and PSD fractions were unaltered (Fig. 3p, q). Taken together, these data suggest that the HFD leads to an impairment in neuronal development and permanent LTP changes in the hippocampus that could contribute to the reduced cognitive performance of HFDfed mice.

We next wondered whether A. muciniphilas treatment, which restores hippocampus-dependent cognitive function in HFD-fed mice, would also rescue hippocampal neuronal development and LTP. We found that $A$. muciniphilas treatment rescued synaptic plasticity (Fig. 3a-i), as well as neuronal development (Fig. $3 \mathrm{j}-\mathrm{q}$ ) in the hippocampus of HFD-fed mice. To be more specifically, using BrdU labeling, A. muciniphilas treatment rescued neuronal proliferation in the DG (Fig. 3j, k). In addition, dendritic morphology and complexity were completely restored by $A$. muciniphilas treatment (Fig. $3 \mathrm{I}-\mathrm{o}$ ), and the expression of GluA1 and GluA2 subunits returned to normal level after $A$. muciniphilas treatment (Fig. 3p, q). Thus, A. muciniphilas treatment prevents HFD-induced neuronal development and LTP impairment in the hippocampus and consequently ameliorates cognitive deficits.

A. muciniphilas treatment reduced microgliosis and inflammation in the hippocampus of HFD-fed mice

We found that HFD-fed mice have a significant deficit in intestinal barrier integrity, as reflected by decreased mRNA level of junction protein in the colon (Fig. 4a), and increased translocation of FITCdextran across the intestinal epithelium, into the circulation (Fig. 4b). Consistent with the leaky gut phenotype, the serum endotoxin level was significantly increased in HFD-fed mice (Fig. 4c), which are consistent with previous study [7]. Gut permeability is commonly associated with an altered immune response. Accordingly, immunohistochemical examination with the microglia marker $\mathrm{lba}-1^{+}$revealed a significant increase in the number of microglia in hippocampus of HFD-fed mice when compared with chow-fed littermates (Fig. 4d, e). Then, we assessed hippocampal proinflammatory cytokines, including TNFa, IL-1 $\beta$, and IL-6. Results revealed that HFD exposure significantly increased the mRNA and protein levels of TNFa, IL-1 1 , and IL- 6 in the hippocampus (Fig. 4f, g), which are consistent with previous report [5]. These results indicate that the aggravation of cognitive impairment of HFD-fed mice may associate with microgliosis and proinflammatory cytokines expression in hippocampus.

We next wondered whether A. muciniphilas treatment, which restores cognitive function in HFD-fed mice, would also inhibit HFD-induced gut permeability, hippocampal microgliosis, and proinflammatory cytokines expression. We found that $A$. muciniphila treatment normalized HFD-induced "leaky gut" (Fig. 4a, b) and metabolic endotoxemia level (Fig. 4c), which is consistent with previous study [20]. Moreover, A. muciniphila treatment completely reversed HFD-induced microgliosis (Fig. 4d, e) and proinflammatory cytokines expression (Fig. 4f, g) in hippocampus.

To directly assess the role of microgliosis and proinflammatory cytokines expression on the hippocampus-dependent cognitive 
A

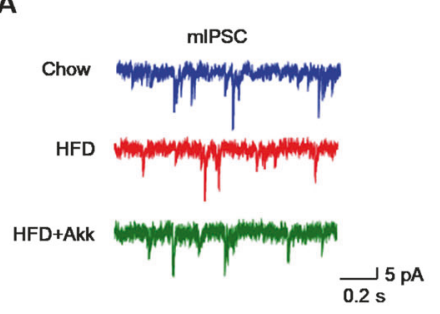

D

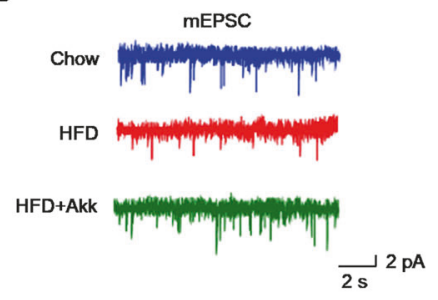

G

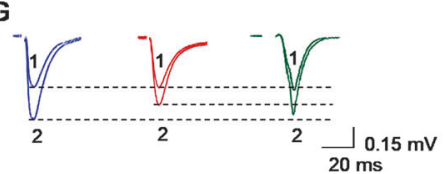

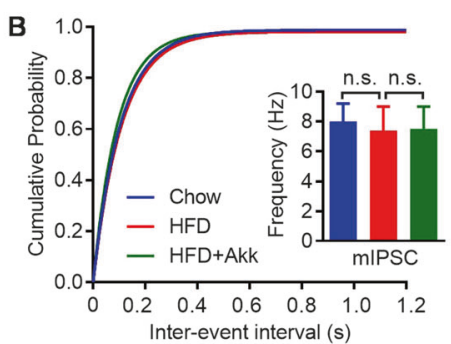

E
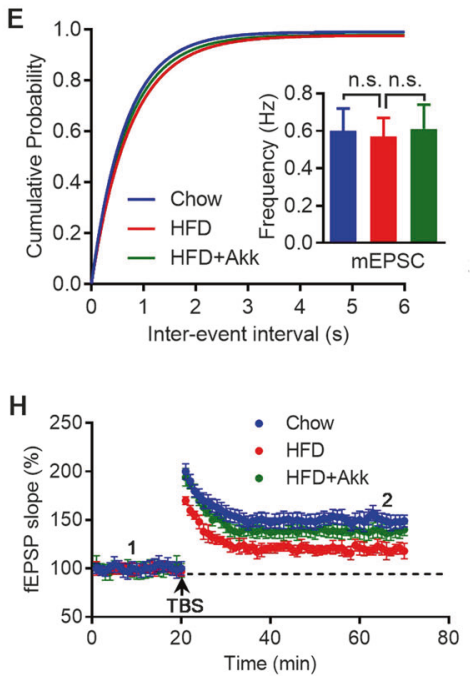

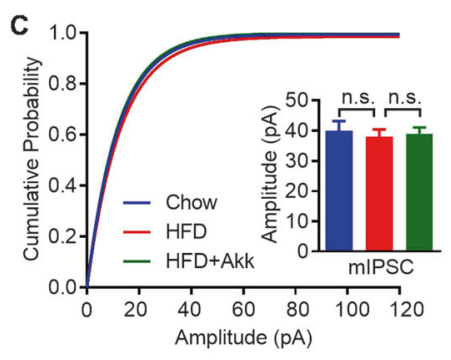

F
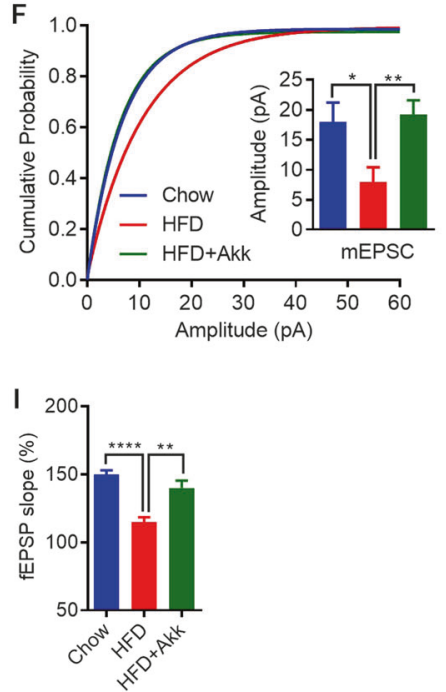
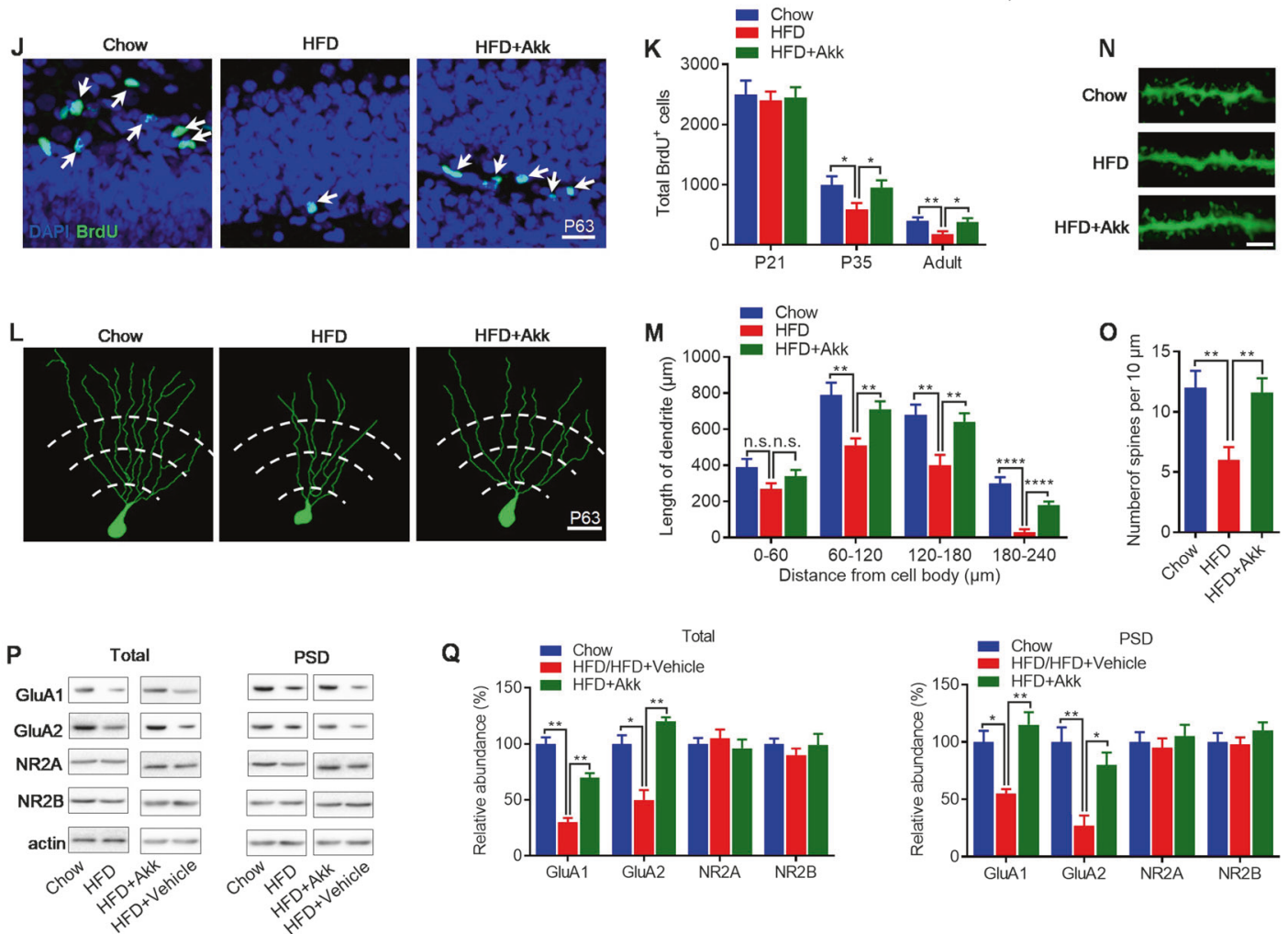

impairment. Microgliosis and proinflammatory cytokines expression were induced in chow-fed mice injected intraperitoneally of cell membrane-derived LPS (Fig. S5a). Results showed that injection of LPS resulted in microgliosis (Fig. S5b, c) and proinflammatory cytokines expression (Fig. S5d) in hippocampus of chow-fed animals, and impaired hippocampus-dependent cognitive function (Fig. S5e-j). Taken together, these findings reveal that microgliosis and proinflammatory cytokines expression may be the strong drivers of hippocampal-dependent cognitive deficits in HFD-fed mice. 
Fig. 3 A. muciniphilas treatment restores synaptic plasticity and neuronal development in the hippocampus of HFD-fed mice. a Representative traces of mIPSCs in CA1 pyramidal neurons from chow-, HFD-, and HFD + Akk-fed mice. Scale bars represent $0.2 \mathrm{~s}, 5 \mathrm{pA}$. b, c Cumulative probability plots of mIPSCs inter-event intervals and histograms of mIPSCs frequency, and amplitude ( $n=15$ neurons of 4 mice/group. d Representative traces of mEPSCs in CA1 pyramidal neurons from chow-, HFD-, and HFD+ Akk-fed mice. Scale bars represent $2 \mathrm{~s}$, 2 pA. e, $\mathbf{f}$ Cumulative probability plots of mEPSCs inter-event intervals and histograms of mEPSCs frequency, and amplitude ( $n=28$ neurons of four mice/group. $g$ Shown on the right were representative traces taken before (1) and 50 min after theta-burst stimulation (TBS) (2), scale bars represent $20 \mathrm{~ms}, 0.15 \mathrm{mV}$. h Impaired LTP at SC-CA1 synapses in the hippocampus of mice. Normalized fEPSP slopes were plotted every 1 min. Arrow denotes LTP induction. i Quantitative analysis of LTP level in I ( $n=9$ slices from 4 mice/group). j Representative images proliferation cells in the subgranular zone (SGZ) of each group at P63, scale bar represents $40 \mu \mathrm{m}$. $\mathbf{k}$ Number of proliferation cells in the SGZ at P21, P35, and P63 were measured $2 \mathrm{~h}$ after BrdU labeling $(n=6 /$ group). I Representative images and corresponding traces of distal granule cells in each group, scale bar represents $20 \mu \mathrm{m}$. $\mathbf{m}$ Dendritic length of distal granule cells in each group ( $n=6 /$ group). $\mathbf{n}, \mathbf{o}$ Spine density in adult (P63) granule cells, scale bar represents $2 \mu \mathrm{m}$. $\mathbf{p}$ Hippocampal tissues were collected from each group and homogenized for western blotting. Shown were representative blots of three independent experiments with similar results. q Quantitative analysis of data in $\mathbf{p}$ ( $n=5 / \mathrm{group}$ ). Band densities of interested proteins were normalized by the loading control $\beta$-actin; values of chow-fed mice were taken as $100 \%$. Means \pm SEM (error bars) are plotted. Statistics were performed with Student's $t$-test or one-way analysis of variance (ANOVA). ${ }^{*} P<0.05,{ }^{* *} P<0.01$, and ****P<0.0001, n.s., not significant

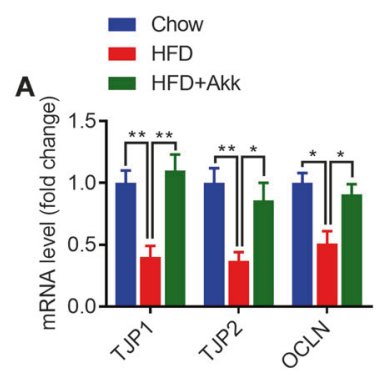

B
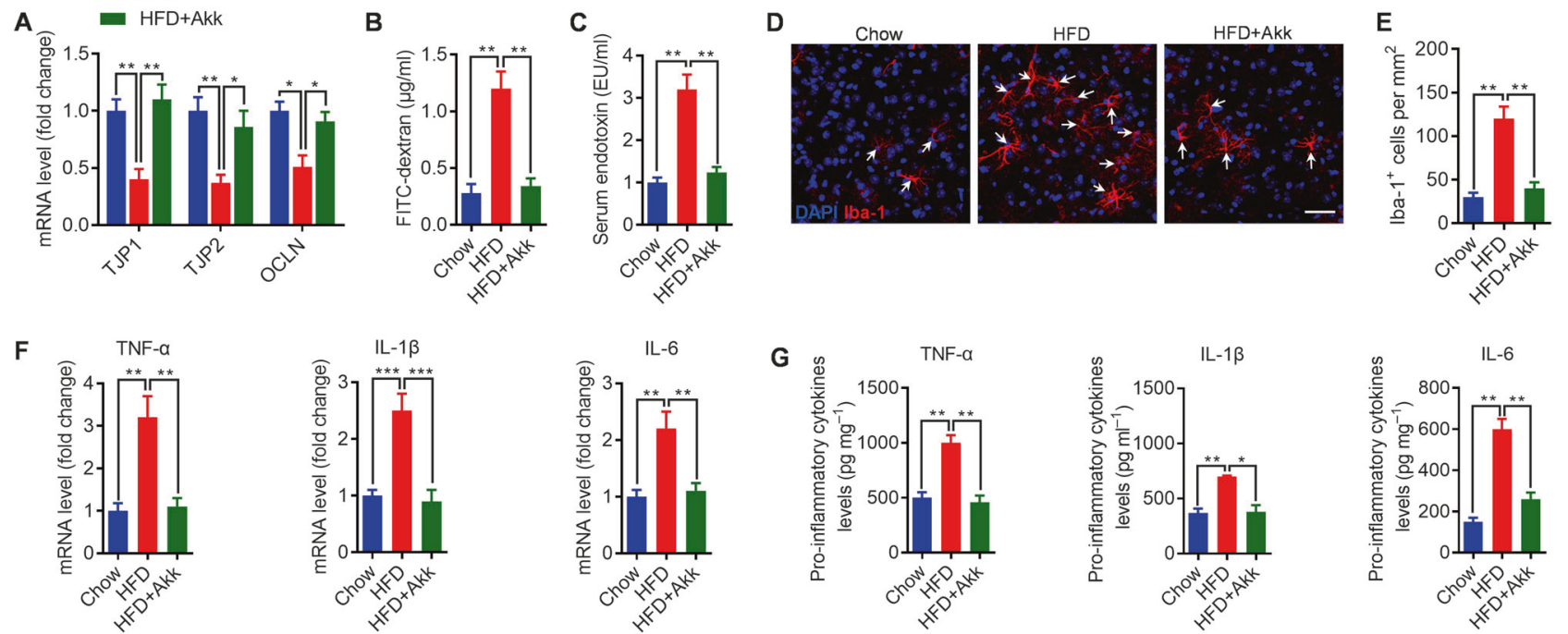

G

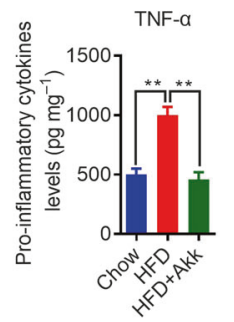

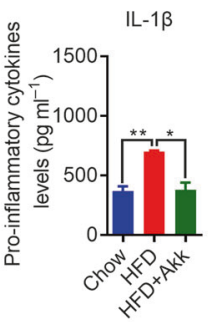

IL-6

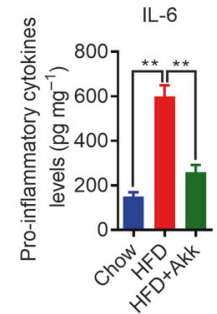

Fig. 4 A. muciniphilas treatment corrects gut barrier deficits and reverses microgliosis and proinflammatory cytokines expression in the hippocampus of HFD-fed mice. a mRNA levels of tight junction component (TJP1, TJP2, and OCLN) in the colon were measured by qRT-PCR. Data for each gene are normalized to chow-fed mice. b Intestinal permeability assay, measuring FITC intensity in serum after oral gavage of FITC dextran. c Serum endotoxin were measured in each group. d Representative confocal micrograph of microglia (Iba- $1^{+}$) in the hippocampus of each group and scale bar, $40 \mu \mathrm{m}$. e Density of lba- $1^{+}$cells in the hippocampus of each group. f mRNA levels of proinflammatory cytokines in the hippocampus of each group. Data for each gene are normalized to chow-fed mice. $\mathbf{g}$ Protein levels of proinflammatory cytokines in the hippocampus of each group. $n=8 /$ group, means \pm SEM (error bars) are plotted. Statistics were performed with Student's $t$-test or one-way analysis of variance (ANOVA). ${ }^{*} P<0.05,{ }^{* *} P<0.01$, and ${ }^{* * *} P<0.00$

TLR4 block or altering the microbiome prevents HFD induced cognitive deficits

LPS activates intracellular signals through the innate immune receptor TLR4, we therefore hypothesized that microglial TLR4 stimulate MyD88-JNK/NF-KB signalling in response to LPS that translocate from the gut lumen to brain-a process strongly influenced by the composition of the gut microbiome. This hypothesis predicts two novel approaches to treat HFD-induced cognitive deficits: TLR4 blockade and manipulation of the microbiome. To directly assess the requirement for TLR4 in HFDinduced hippocampal-dependent cognitive decline, TAK-242 (a small-molecule TLR4 antagonist that binds the intracellular domain of TLR4 and blocks signal transduction) [35] and LPS-RS (a hypo-acetylated LPS derived from Rhodobacter sphaeroides that competitively antagonizes TLR4) [36] were used to blockade TLR4 signal pathway (Fig. 5a). We found that TAK-242 or LPS-RS treatment inhibits the proinflammatory cytokines expression in hippocampus of HFD-fed animals (Fig. 5b). Results showed that treatment of HFD-fed mice with TAK-242 rescued the hippocampal-dependent cognitive deficits in HFD-fed mice (Fig. 5c-h). Consistently, treatment of HFD-fed mice with LPS-RS conferred a great hippocampal-dependent cognitive function improvement compared with vehicle-treated littermates (Fig. 5i-n). However, TAK-242 or LPS-RS treatment have no effects on cognitive functions (Fig. S6a-g) and body mass composition (Fig. S6h-o) of chow-fed mice. Overall, these results confirm the essential role of TLR4 signalling in HFD induced hippocampaldependent cognitive deficits and suggest that TLR4 antagonists may be effective therapies.

To test the effect of deliberate microbiome manipulation on cognitive deficits in HFD-fed mice, we used antibiotics to reset the microbiome. Broad-spectrum antibiotics were administered in HFD-fed mice (Fig. S7a). As expected, antibiotic treatment significantly reduced the total gut bacterial load and alpha diversity (Fig. S7b-d). Abx-treated mice maintained lower bodyweights and had lower body fat percentages and higher leanbody-mass percentages when compared with vehicle-treated littermates (Fig. S7e-h). Furthermore, Abx treatment inhibits 

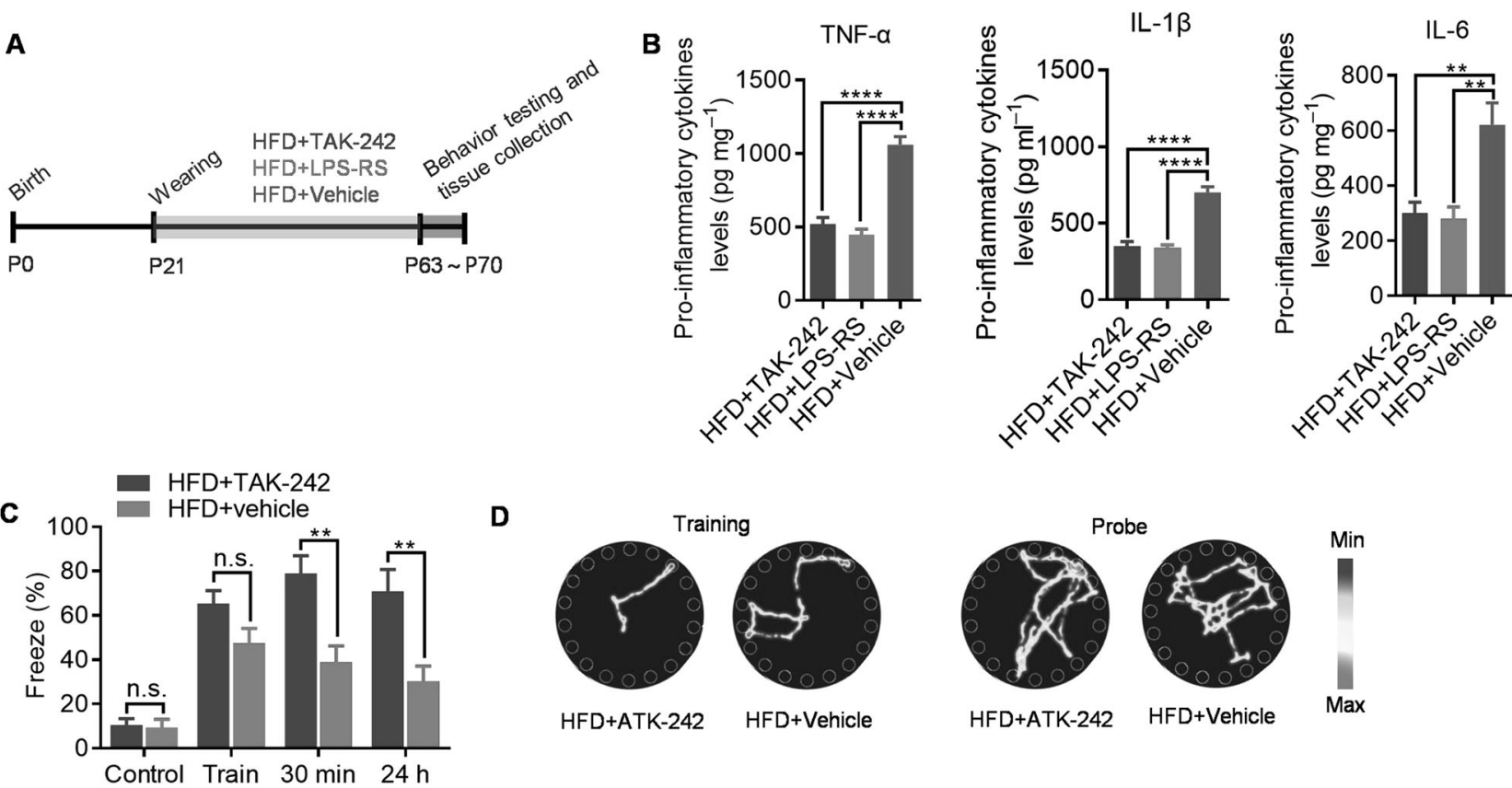

D
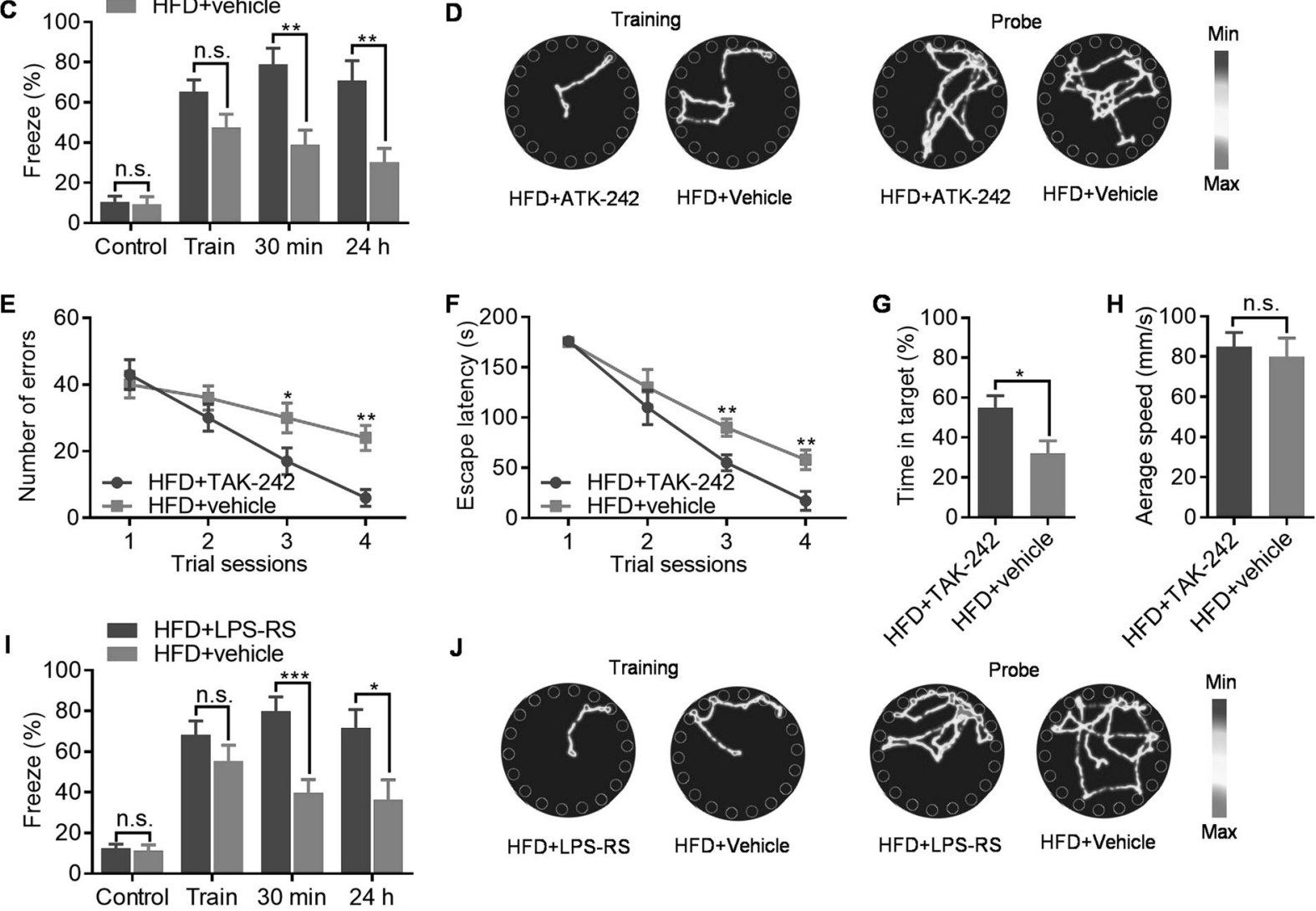

$\mathbf{J}$
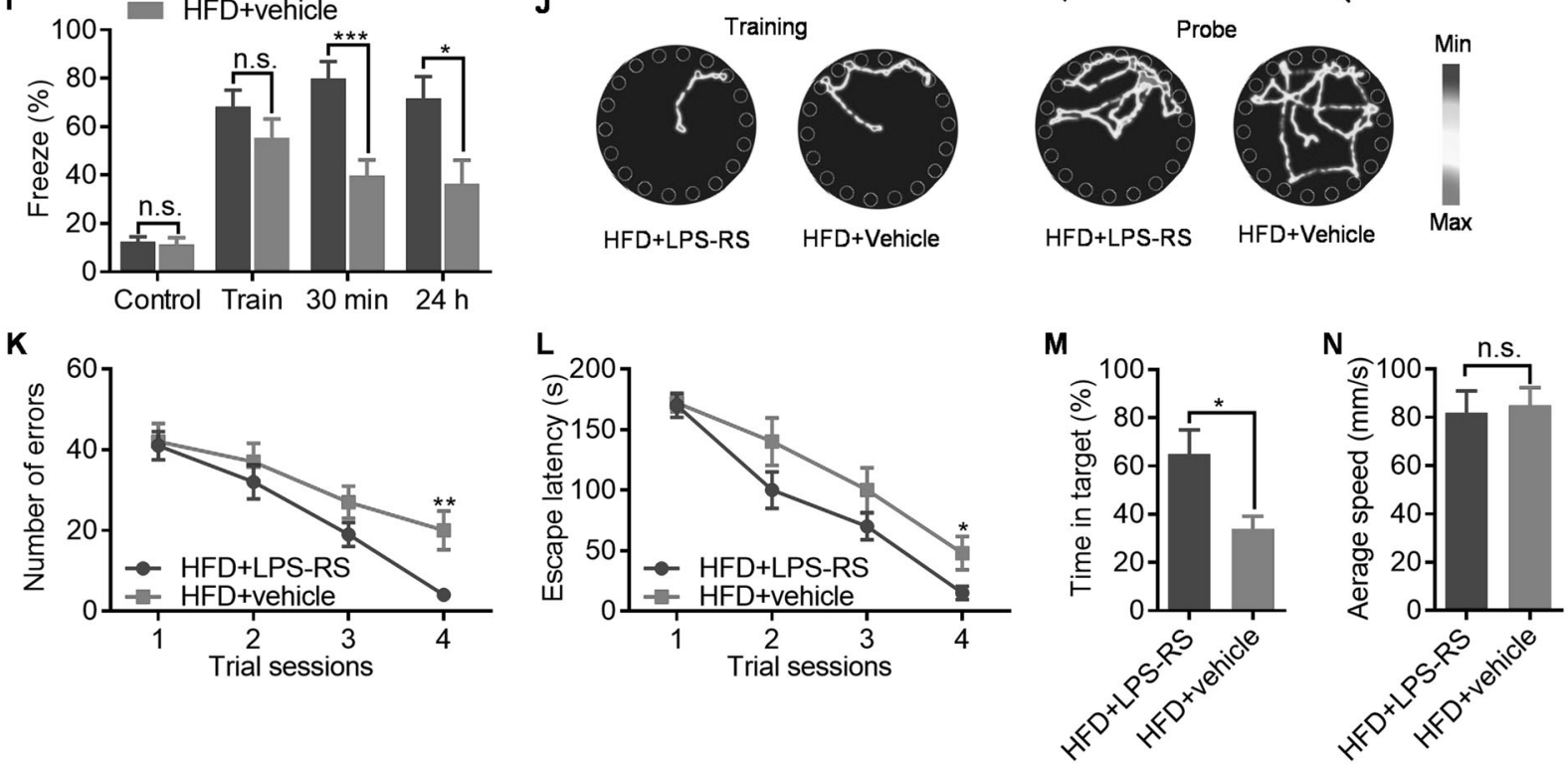

microgliosis (Fig. S7i, j) and proinflammatory cytokines expression (Fig. S7k) in hippocampus of HFD-fed animals, in association with an improved hippocampal-dependent cognitive function in HFDfed mice (Fig. S7l-q). Collectively, these findings provide further evidence that qualitative changes in the bacterial microbiome can alter HFD-induced hippocampal-dependent cognitive decline.

\section{DISCUSSION}

In the present study, we demonstrate that HFD influences hippocampus development and hippocampal-dependent cognitive function in mice. These effects are associated with dysregulation of gut microbiota. In addition, we found that $A$. muciniphila treatment is sufficient to rescue HDF-induced 
Fig. 5 Preventing HFD-induced cognitive deficits by TLR4 antagonism. a Schematic of TLR4 antagonism experiment. b Protein levels of proinflammatory cytokines in the hippocampus of TAK-242-, LPS-RS-, and vehicle-treated mice. c Percent of freezing in the contextual fearconditioning test. $\mathbf{d}$ Representative traces of TAK-242- and vehicle-treated mice in Barnes circular maze test. e Number of errors in the training session of Barnes circular maze. $\mathbf{f}$ Escape latency in the training session of Barnes circular maze test. $\mathbf{g}$ Time in the target quadrant during the probe trial on the fifth day of Barnes circular maze test. $\mathbf{h}$ Average speed during the probe trial on the fifth day of Barnes circular maze test. $\mathbf{i}$ Percent of freezing in the contextual fearconditioning test. $\mathbf{j}$ Representative traces of LPS-RS- and vehicle-treated mice in Barnes circular maze test. k Number of errors in the training session of Barnes circular maze. I Escape latency in the training session of Barnes circular maze test. $\mathbf{m}$ Time in the target quadrant during the probe trial on the fifth day of Barnes circular maze test. $\mathbf{n}$ Average speed during the probe trial on the fifth day of Barnes circular maze test. $n=8$ /group, means \pm SEM (error bars) are plotted. Statistics were performed with Student's $t$-test or oneway analysis of variance (ANOVA). ${ }^{*} P<0.05$, ${ }^{* *} P<0.01,{ }^{* * *} P<0.001,{ }^{* * *} P<0.0001$, and n.s., not significant

cognitive impairment, which indicates that $A$. muciniphila treatment is a potential probiotic therapy for obesity associated with cognitive dysfunction during early life. Our findings are relevant not only to the understanding of etiology of HFD on the developmental progression of hippocampus, but also demonstrate the importance of gut microbiota for the normal brain functions.

Compelling evidence suggests there is an association between HFD, gut microbiota, and body weight regulation [3, 4, 37, 38]. Obesity in childhood and adolescence could have a particularly relevant impact since this is a critical periods for neurodevelopment and neuronal plasticity [34, 39, 40], where HFD can significantly alter brain functions, behaviors, and mood states in adulthood [41]. Here, we demonstrated that animals fed HFD show great potentially pathological changes in hippocampus, including impaired synaptic plasticity, reduced neuron proliferation, and atrophic dendritic spines.

A variety of factors could contribute to the HFD-induced cognitive function abnormalities. HFD has been shown to alter the gut microbiome and individuals diagnosed with obesity can copresent dysbiosis of the gut microbiota $[4,38]$. The associations established between obesity and cognitive impairment in epidemiological and experimental studies point to shared contributing factors, and pathophysiological mechanisms [4]. These associations could be related to dietary induced alterations in the intestinal microbiota that, in turn, may contribute to (neuro) inflammation and dysregulation of the neuroendocrine system associated with obesity comorbid with cognitive impairments $[4,42,43]$. We showed that HFD induces alterations of gut microbiota, particularly by depleting A. muciniphila. A. muciniphila within the mucus layer is implicated in the control of host mucus turnover, which improves gut barrier function and is linked to obesity [20, 24, 25]. HFD-fed animals are reported displays GI abnormalities, including increased intestinal permeability or "leaky gut" $[7,44,45]$, which leads to translocation of bacterial LPS into the blood and producing inflammation [7, 44, 45]. In HFD-induced obesity, endotoxemia has been linked with alterations of gut microbiota and increased intestinal permeability [19]. Currently, obesity and the related cognitive decline are associated with microbiota alterations and low-grade systemic and central inflammation, which contribute to interruption of the gut-brain axis $[3,19]$. This hypothesis has been confirmed to some extent in this study, which investigated the effectiveness of $A$. muciniphila treatment in both attenuating obesity and associated cognitive disorders.

It has been reported that activated microglial cells release proinflammatory cytokines TNF- $a, I L-1 \beta$, and IL- 6 , which negatively regulate cognitive functions [12-14]. Our findings demonstrated that HFD promoted the expression of lba- 1 in hippocampus suggesting an excessive microgliasis. Hippocampal inflammation is highly associated with recognition memory impairment [14]. Therefore, $A$. muciniphila treatment that prevent high endotoxin level and inhibit hippocampal microgliosis and inflammation may contribute to spatial learning, and memory restoration. TLR4 is the receptor for LPS and expressed in microglia, astrocytes, and neurons in the brain [46]. Activation of TLR4 signaling inhibits neurogenesis, while deficiency of TLR4 dramatically ameliorates neuro-inflammation in the hippocampus of mice [46]. In this study, A. muciniphila treatment prevented the activation of the TLR4 inflammatory signaling pathway and an overexpression of proinflammatory cytokines in the hippocampus induced by a HFD feeding. Besides, TAK-242 or LPS-RS treatment effectively reduce HFD-induced cognitive deficits, confirming TLR4 as a 'druggable' target for obesity associated cognitive disorders. Meanwhile, manipulation of gut microbiome-host interactions is also an exciting potential strategy to treat a life-long disease such as HFDinduced cognitive deficits.

\section{CONCLUSIONS}

In conclusion, this study demonstrated that $A$. muciniphila reinstalls and restores cognitive function in HFD-induced obese mice. These beneficial effects are accompanied by a reduction of hippocampal microgliosis. The behavioral and neurochemical improvements suggest that $A$. muciniphila could be a promising strategy to improve gut-brain axis for the prevention of HFDinduced obesity and associated cognitive decline. However, further translational and functional studies are needed to progress in the identification of molecular targets/pathways that could be favorable modulated by microbiota-based interventions to help reduce obesity associated complications.

\section{FUNDING AND DISCLOSURE}

This study was supported by grants from the National Natural Science Foundation of China (81703841 to Y.J.Y.), China Postdoctoral Science Foundation (2018T110955 to Y. J.Y., 2017 M612929 to Y.J.Y.), Sichuan Province Postdoctoral Science Foundation (008072001 to Y.J.Y.), and Chengdu University of TCM Science Foundation (ZRQN1718 to Y.J.Y., BSH2018003 to Y.J.Y.). The authors declare no competing interests.

\section{ADDITIONAL INFORMATION}

Supplementary Information accompanies this paper at (https://doi.org/10.1038/ s41386-019-0437-1).

Publisher's note: Springer Nature remains neutral with regard to jurisdictional claims in published maps and institutional affiliations.

\section{REFERENCES}

1. Vainik U, Baker TE, Dadar M, Zeighami Y, Michaud A, Zhang Y, et al. Neurobehavioral correlates of obesity are largely heritable. Proc Natl Acad Sci USA. 2018;115:9312-7.

2. Bocarsly ME, Fasolino M, Kane GA, LaMarca EA, Kirschen GW, Karatsoreos IN, et al. Obesity diminishes synaptic markers, alters microglial morphology, and impairs cognitive function. Proc Natl Acad Sci USA. 2015;112:15731-6.

3. Sellbom KS, Gunstad J. Cognitive function and decline in obesity. J Alzheimers Dis. 2012;30(Suppl 2):S89-95.

4. Agusti A, Garcia-Pardo MP, Lopez-Almela I, Campillo I, Maes M, Romani-Perez M, et al. Interplay between the gut-brain axis, obesity, and cognitive function. Front Neurosci. 2018;12:155.

5. Boitard C, Cavaroc A, Sauvant J, Aubert A, Castanon N, Laye S, et al. Impairment of hippocampal-dependent memory induced by juvenile high-fat diet intake is 
associated with enhanced hippocampal inflammation in rats. Brain Behav Immun. 2014;40:9-17.

6. Valcarcel-Ares MN, Tucsek Z, Kiss T, Giles CB, Tarantini S, Yabluchanskiy A, et al. Obesity in aging exacerbates neuroinflammation, dysregulating synaptic function-related genes, and altering eicosanoid synthesis in the mouse hippocampus: potential role in impaired synaptic plasticity and cognitive decline. J Gerontol A Biol Sci Med Sci. 2019;74:290-8.

7. Wu TR, Lin CS, Chang CJ, Lin TL, Martel J, Ko YF, et al. Gut commensal Parabacteroides goldsteinii plays a predominant role in the anti-obesity effects of polysaccharides isolated from Hirsutella sinensis. Gut. 2019;68:248-62.

8. Skelly DT, Griffin EW, Murray CL, Harney S, O'Boyle C, Hennessy E, et al. Acute transient cognitive dysfunction and acute brain injury induced by systemic inflammation occur by dissociable IL-1-dependent mechanisms. Mol Psychiatr. 2018. https://doi.org/10.1038/s41380-018-0075-8.

9. Aguzzi A, Barres BA, Bennett ML. Microglia: scapegoat, saboteur, or something else? Science. 2013;339:156-61.

10. Stephan AH, Barres BA, Stevens B. The complement system: an unexpected role in synaptic pruning during development and disease. Annu Rev Neurosci. 2012;35:369-89.

11. Schafer DP, Lehrman EK, Kautzman AG, Koyama R, Mardinly AR, Yamasaki R, et al. Microglia sculpt postnatal neural circuits in an activity and complementdependent manner. Neuron. 2012;74:691-705.

12. Deczkowska A, Keren-Shaul H, Weiner A, Colonna M, Schwartz M, Amit I, et al. Disease-associated microglia: a universal immune sensor of neurodegeneration. Cell. 2018;173:1073-81.

13. Song WM, Colonna M. The identity and function of microglia in neurodegeneration. Nat Immunol. 2018;19:1048-58.

14. Hickman S, Izzy S, Sen P, Morsett L, El Khoury J. Microglia in neurodegeneration. Nat Neurosci. 2018;21:1359-69.

15. De Luca SN, Ziko I, Sominsky L, Nguyen JC, Dinan T, Miller AA, et al. Early life overfeeding impairs spatial memory performance by reducing microglial sensitivity to learning. J Neuroinflammation. 2016;13:112.

16. Wadhwa M, Prabhakar A, Ray K, Roy K, Kumari P, Jha PK, et al. Inhibiting the microglia activation improves the spatial memory and adult neurogenesis in rat hippocampus during $48 \mathrm{~h}$ of sleep deprivation. J Neuroinflamm. 2017; 14:222

17. Sato K. Effects of microglia on neurogenesis. Glia. 2015;63:1394-405.

18. Bruce-Keller AJ, Salbaum JM, Luo M, Blanchard Et, Taylor CM, Welsh DA, et al. Obese-type gut microbiota induce neurobehavioral changes in the absence of obesity. Biol Psychiatry. 2015;77:607-15.

19. Guillemot-Legris O, Muccioli GG. Obesity-induced neuroinflammation: beyond the hypothalamus. Trends Neurosci. 2017;40:237-53.

20. Everard A, Belzer C, Geurts L, Ouwerkerk JP, Druart C, Bindels LB, et al. Cross-talk between Akkermansia muciniphila and intestinal epithelium controls diet-induced obesity. Proc Natl Acad Sci USA. 2013;110:9066-71.

21. Collado MC, Derrien M, Isolauri E, de Vos WM, Salminen S. Intestinal integrity and Akkermansia muciniphila, a mucin-degrading member of the intestinal microbiota present in infants, adults, and the elderly. Appl Environ Microbiol. 2007;73:7767-70.

22. Derrien M, Collado MC, Ben-Amor K, Salminen S, de Vos WM. The Mucin degrader Akkermansia muciniphila is an abundant resident of the human intestinal tract. Appl Environ Microbiol. 2008;74:1646-8.

23. Reunanen J, Kainulainen V, Huuskonen L, Ottman N, Belzer C, Huhtinen $\mathrm{H}$, et al. Akkermansia muciniphila Adheres to enterocytes and strengthens the integrity of the epithelial cell layer. Appl Environ Microbiol. 2015;81:3655-62.

24. Derrien $M$, Vaughan EE, Plugge CM, de Vos WM. Akkermansia muciniphila gen. nov., sp. nov., a human intestinal mucin-degrading bacterium. Int J Syst Evol Microbiol. 2004;54:1469-76.

25. Belzer C, de Vos WM. Microbes inside-from diversity to function: the case of Akkermansia. Isme j. 2012;6:1449-58.
26. Plovier H, Everard A, Druart C, Depommier C, Van Hul M, Geurts L, et al. A purified membrane protein from Akkermansia muciniphila or the pasteurized bacterium improves metabolism in obese and diabetic mice. Nat Med. 2017;23:107-13.

27. Grander C, Adolph TE, Wieser V, Lowe P, Wrzosek L, Gyongyosi B, et al. Recovery of ethanol-induced Akkermansia muciniphila depletion ameliorates alcoholic liver disease. Gut. 2018:67:891-901.

28. Hanninen A, Toivonen R, Poysti S, Belzer C, Plovier H, Ouwerkerk JP, et al. Akkermansia muciniphila induces gut microbiota remodelling and controls islet autoimmunity in NOD mice. Gut. 2018;67:1445-53.

29. Olson CA, Vuong HE, Yano JM, Liang QY, Nusbaum DJ, Hsiao EY. The Gut microbiota mediates the anti-seizure effects of the ketogenic Diet. Cell. 2018;173:1728-41.

30. Chevalier C, Stojanovic O, Colin DJ, Suarez-Zamorano N, Tarallo V, Veyrat-Durebex $C$, et al. ut microbiota orchestrates energy homeostasis during cold. Cell. 2015;163:1360-74

31. Liu B, Zupan B, Laird E, Klein S, Gleason G, Bozinoski M, et al. Maternal hematopoietic TNF, via milk chemokines, programs hippocampal development and memory. Nat Neurosci. 2014;17:97-105.

32. Han S, Tai C, Westenbroek RE, Yu FH, Cheah CS, Potter GB, et al. Autistic-like behaviour in Scn1 $\mathrm{a}^{+/-}$mice and rescue by enhanced GABA-mediated neurotransmission. Nature. 2012:489:385-90.

33. Buffington SA, Di Prisco GV, Auchtung TA, Ajami NJ, Petrosino JF, Costa-Mattioli M. Microbial reconstitution reverses maternal diet-induced social and synaptic deficits in offspring. Cell. 2016;165:1762-75.

34. Boitard C, Etchamendy N, Sauvant J, Aubert A, Tronel S, Marighetto A, et al. Juvenile, but not adult exposure to high-fat diet impairs relational memory and hippocampal neurogenesis in mice. Hippocampus. 2012;22:2095-100.

35. Matsunaga N, Tsuchimori N, Matsumoto T, li M. TAK-242 (resatorvid), a smallmolecule inhibitor of Toll-like receptor (TLR) 4 signaling, binds selectively to TLR4 and interferes with interactions between TLR4 and its adaptor molecules. Mol Pharmacol. 2011;79:34-41.

36. Coats SR, Pham TT, Bainbridge BW, Reife RA, Darveau RP. MD-2 mediates the ability of tetra-acylated and penta-acylated lipopolysaccharides to antagonize Escherichia coli lipopolysaccharide at the TLR4 signaling complex. J Immunol. 2005; 175:4490-8.

37. Turnbaugh PJ, Backhed F, Fulton L, Gordon ال Diet-induced obesity is linked to marked but reversible alterations in the mouse distal gut microbiome. Cell Host Microbe. 2008;3:213-23.

38. Bliss ES, Whiteside E. The gut-brain axis, the human gut microbiota and their integration in the development of obesity. Front Physiol. 2018;9:900.

39. Spear LP. The adolescent brain and age-related behavioral manifestations. Neurosci Biobehav Rev. 2000;24:417-63.

40. Monda V, La Marra M, Perrella R, Caviglia G, lavarone A, Chieffi S, et al. Obesity and brain illness: from cognitive and psychological evidences to obesity paradox. Diabetes Metab Syndr Obes. 2017;10:473-9.

41. Kundakovic M, Champagne FA. Early-life experience, epigenetics, and the developing brain. Neuropsychopharmacol. 2015;40:141-53.

42. Wang S, Huang XF, Zhang P, Newell KA, Wang H, Zheng K, et al. Dietary teasaponin ameliorates alteration of gut microbiota and cognitive decline in dietinduced obese mice. Sci Rep. 2017;7:12203.

43. Wang S, Huang XF, Zhang P, Wang H, Zhang Q, Yu S, et al. Chronic rhein treatment improves recognition memory in high-fat diet-induced obese male mice. J Nutr Biochem. 2016;36:42-50.

44. Cani PD, Bibiloni R, Knauf C, Waget A, Neyrinck AM, Delzenne NM, et al. Changes in gut microbiota control metabolic endotoxemia-induced inflammation in highfat diet-induced obesity and diabetes in mice. Diabetes. 2008:57:1470-81.

45. Cani PD, Possemiers S, Van de Wiele T, Guiot Y, Everard A, Rottier O, et al. Changes in gut microbiota control inflammation in obese mice through a mechanism involving GLP-2-driven improvement of gut permeability. Gut. 2009:58:1091-103.

46. Trotta T, Porro C, Calvello R, Panaro MA. Biological role of Toll-like receptor-4 in the brain. J Neuroimmunal. 2014;268:1-12. 